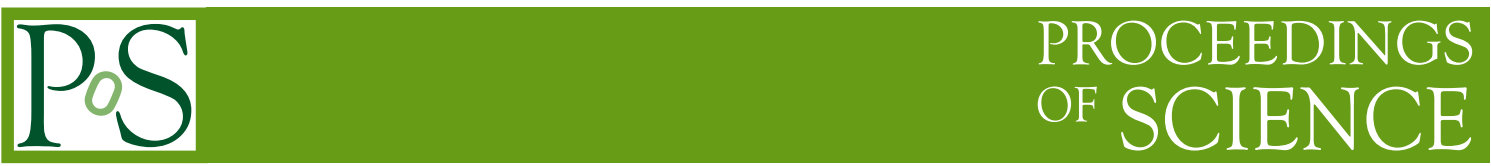

\title{
Measurements of jets in heavy ion collisions
}

\author{
Christine Nattrass* \\ University of Tennessee, Knoxville \\ E-mail: christine.nattrasseutk.edul
}

The Quark-Gluon Plasma (QGP) is created in high energy heavy ion collisions at the Relativistic Heavy Ion Collider (RHIC) and the Large Hadron Collider (LHC). This medium is transparent to electromagnetic probes but nearly opaque to colored probes. Hard partons produced early in the collision fragment and hadronize into jets. The partons lose energy as they traverse the medium. Most of the lost energy is still correlated with the parent parton, contributing to particle production at larger angles and lower momenta relative to the parent parton than in proton-proton collisions. This partonic energy loss can be measured through several observables, each of which give different insights into the degree and mechanism of energy loss. The measurements to date are summarized and the path forward is discussed.

12th International Workshop on High-pT Physics in the RHIC/LHC era,

October 2-5, 2017

University of Bergen, Norway

${ }^{*}$ Speaker. 


\section{Introduction}

A dense, hot liquid called the Quark Gluon Plasma (QGP) is formed in high energy heavy ion collisions [1-4]. Hard partons scattered early in the collision traverse the medium and fragment into collimated sprays of particles called jets. In principle, partonic energy loss can be used to deduce the properties of the medium. The ultrarelativistic heavy ion community has accumulated high quality data from the Relativistic Heavy Ion Collider (RHIC) and the Large Hadron Collider (LHC) for nearly two decades. The wealth of data has given us many insights into partonic energy loss in the QGP, but there is still work to be done. I first briefly review experimental results and then discuss some of the challenges the community must address moving forward.

\section{What we have learned}

As partons traverse the medium, they lose energy either through collisional energy loss or gluon bremsstrahlung. As a result, the parton shower is broader and the average momentum of final state partons is lower than in $p+p$ collisions. This process is frequently referred to as, "jet quenching." We recently reviewed the experimental evidence for jet quenching in [5]. The term jet quenching may be misleading, since it implies that the energy from the hard parton is no longer distinguishable from the background. Partonic energy loss results in fewer final state hadrons which carry a high fraction of the parent parton's momentum and therefore a suppression of high momentum final state hadrons. Low momentum hadrons are enhanced and, unless the energy from the jet reaches complete equilibrium with the medium, retain their spatial correlations with the jet axis.
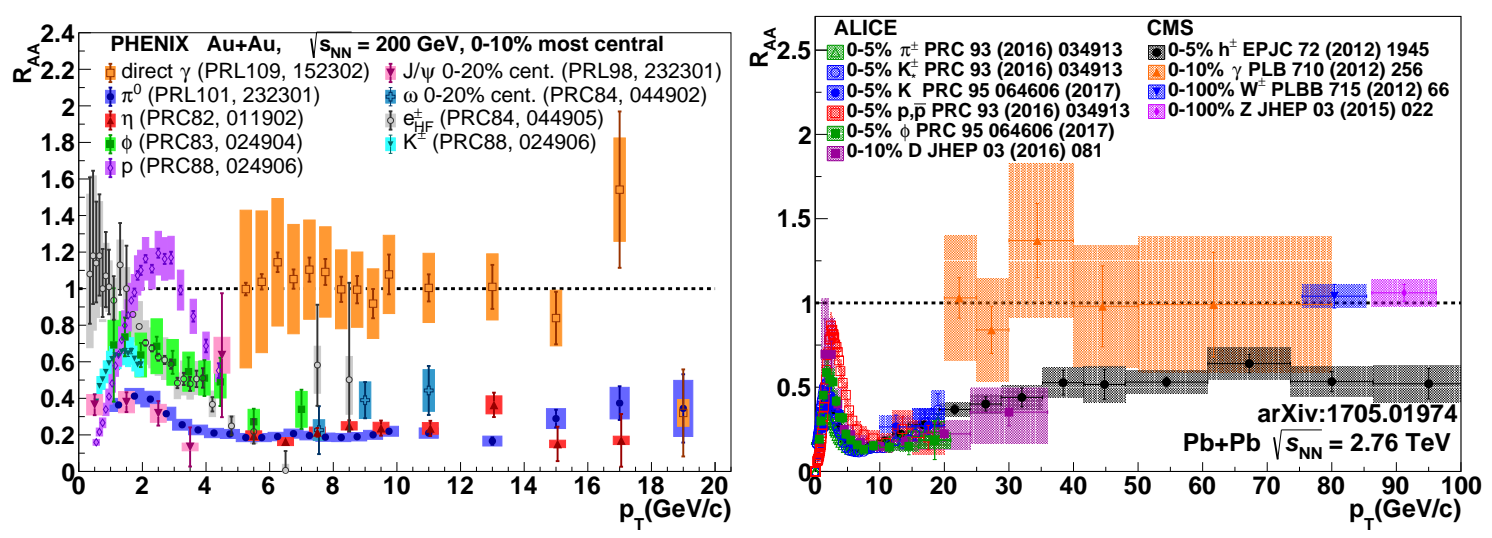

Figure 1: Compilation of $R_{A A}$ from PHENIX (left) and the LHC (right) from [5].

The most straightforward way to quantify jet quenching is through the measurement of single hadrons at high momentum. The scaled ratio of the transverse momentum spectra of single hadrons, called the nuclear modification, is defined as

$$
R_{A A}=\frac{\sigma_{N N}}{\left\langle N_{b i n}\right\rangle} \frac{d^{2} N_{A A} / d p_{T} d \eta}{d^{2} \sigma_{p p} / d p_{T} d \eta}
$$




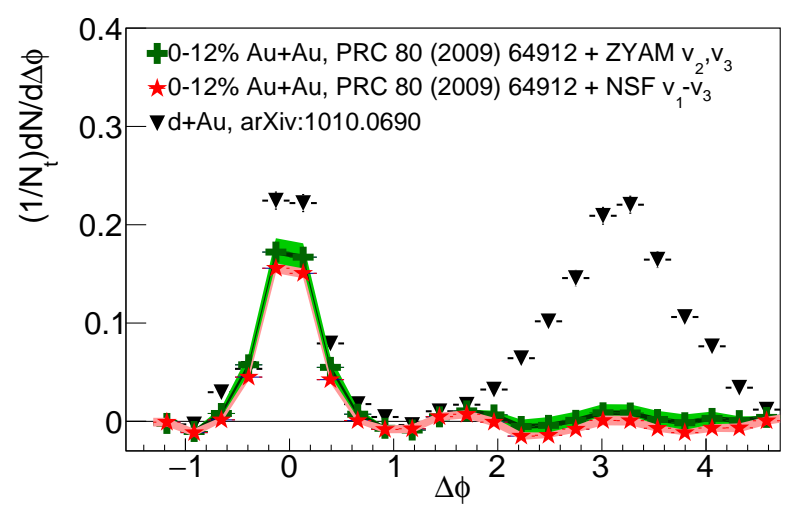

Figure 2: Dihadron correlations with $4<p_{T}^{\mathrm{t}}<6 \mathrm{GeV} / c$ and $2<p_{T}^{\mathrm{a}}<4 \mathrm{GeV} / c$ from minimum bias $d+\mathrm{Au}$ collisions at $\sqrt{s_{\mathrm{NN}}}=200 \mathrm{GeV}$ from [6] and 0-10\% collisions from [7] reanalyzed using both the Near-Side Fit method described in [8] and the ZYAM method using $v_{2}$ from [7] and $v_{3}$ from [9] from a reanalysis of [10] from in [11].

where $\eta$ is the pseudorapidity, $p_{T}$ is the transverse momentum, $\left\langle N_{b i n}\right\rangle$ is the average number of binary nucleon-nucleon collisions for a given range of impact parameter, and $\sigma_{N N}$ is the integrated nucleon-nucleon cross section. $R_{A A}$ has been measured to high precision for several different hadrons, shown in figure 1 for both $\sqrt{s_{\mathrm{NN}}}=200 \mathrm{GeV}$ and $\sqrt{s_{\mathrm{NN}}}=2.76 \mathrm{TeV}$. Substantial suppression is observed for all hadrons and for leptons from the decays of heavy flavor hadrons. The JET collaboration compared $R_{A A}$ to models systematically in order to constrain the jet transport coefficient $\hat{q}=Q^{2} / L$ [12], the transverse momentum lost to the medium $(Q)$ squared divided by the path length traversed by the parton. This calculation remains the best example of constraints on QGP properties by studies of jet quenching.

Correlations between high momentum hadron pairs played a pivotal role in our understanding of partonic energy loss as well, with nearly forty papers published by experimental collaborations on dihadron correlations and over 760 citations for the first paper demonstrating that parton pairs $180^{\circ}$ away from the trigger hadron are suppressed [10]. Since the publication of the original observation of jet quenching using dihadron correlations, we learned that a key component of the background was omitted, triangular flow, $v_{3}$. These data were reanalyzed in [11] using our current knowledge of the background, shown in figure 2. Fortunately for the field, the data are qualitatively consistent with the earlier analysis.

Fully reconstructed jets have confirmed the picture of jet quenching developed in the RHIC era based on single hadron spectra and dihadron correlations. Partonic energy loss has been observed using di-jet asymmetries [13], $\gamma$-hadron correlations [14], $\gamma$-jet correlations [15],hadron-jet correlations [16], and azimuthal anisotropies of jet spectra [17]. The broadening and softening of the fragmentation function has been observed using dihadron correlations [18], $\gamma$-hadron correlations [14], jet-hadron correlations [19], and fragmentation functions [20]. This qualitatively confirms the picture of partonic energy loss through bremsstrahlung and collisional energy loss. The amount of quantitative information about the properties of the plasma is unfortunately still rather limited.

In an effort to best determine the properties of the medium from measurements of jets, the field 
recently investigating new observables which may be more sensitive to the jet structure [21-24]. The idea is that our traditional observables may not be the most sensitive to the physics we are trying to learn.

\section{What we should have learned}

Even in elementary collisions where there is little or no background, jets are ambiguously defined, even on the parton level. If a gluon is emitted at a small angle relative to a parent parton, it is unclear if this parton is part of the jet or a jet itself. The choice is ultimately arbitrary. Early measurements of jets used experimental algorithms which could not be reproduced reliably in theoretical calculations, for instance because they required a high momentum seed. This problem was solved by a combination of the Snowmass Accord [25], an agreement that good jet finders should be both theoretically and experimentally robust, and the development of a number of jet finding algorithms which meet these requirements [26].

The presence of a large, fluctuating background in heavy ion collisions has led to the development of several background subtraction and suppression algorithms, most of which include kinematic requirements on jet constituents. None of the measurements of jet spectra in heavy ion collisions meet the requirements laid out in the Snowmass Accord. The impact of the background suppression and subtraction is usually corrected using PYTHIA [27] embedded into heavy ion collisions. This makes the inherent assumption that PYTHIA jets are a good description of jets in heavy ion collisions, even though we know that jets are modified through their interactions of the medium. The modifications may not make an important impact on the experimental corrections, but it is difficult quantify how interactions with the medium affect these corrections because there are no models which incorporate all aspects of both the background and the jet signal.

Furthermore, experimental techniques to suppress and subtract background favor jets which have hard, tightly collimated fragments. Most measurements therefore have a survivor bias and therefore can only provide a partial picture. This can help explain some apparently contradictory results, such as the tension between ALICE [28] and ATLAS [29] jet $R_{A A}$ at low momenta. The appropriate treatment of background and the impact of bias on measurements is seldom discussed.

\section{How we move forward}

The JETSCAPE collaboration was formed to address the need for Monte Carlo models incorporating both realistic background and approaches to jet quenching. After incorporating jet quenching into Monte Carlo models, a Bayesian analysis will be done to determine the model parameters which best describe the data, similar to analyses in [30,31] but incorporating measurements of jets. This requires a detailed understanding of the measurements themselves, including the treatment of background.

In [5] we proposed a meeting to discuss the treatment of background in theory and experiment. This workshop will take place June 25-27, 2018 at BNL with the aim of reaching an agreement on reasonable ways to treat background consistently in both theoretical calculations and experimental measurements. The combination of these efforts will allow a deeper understanding of the medium and provide guidance for future measurements. The development of realistic Monte Carlos by 
JETSCAPE will also allow realistic studies to determine which, if any, of the new observables currently being investigated are both robust in the presence of background and sensitive to the properties of the medium. Without a realistic and honest discussion about the treatment of background, however, the quantitative limits measurements of jets can provide on properties of the medium will be severely limited.

\section{Conclusions}

Over the last two decades, the wealth of data from RHIC and the LHC have qualitatively confirmed the picture of partonic energy loss in the medium through bremsstrahlung and collisional energy loss. Systematic comparisons to single particle $R_{A A}$ provided constraints on $\hat{q}$. The community needs to constrain the properties of the medium better using the wealth of measurements. This requires both the development and use of realistic Monte Carlo models and a thoughtful, careful treatment of background in both experimental measurements.

\section{Acknowledgements}

I am grateful to Redmer Bertens for useful comments on the manuscript and Megan Connors, Rosi Reed, and Sevil Salur for many useful discussions. This work was supported by funding from the Division of Nuclear Physics of the U.S. Department of Energy under Grant No. DE-FG0296ER40982.

\section{References}

[1] PHENIX Collaboration, K. Adcox et al., "Formation of dense partonic matter in relativistic nucleus nucleus collisions at RHIC: Experimental evaluation by the PHENIX collaboration," Nucl. Phys. A757 (2005) 184-283.

[2] STAR Collaboration, J. Adams et al., "Experimental and theoretical challenges in the search for the quark gluon plasma: The STAR collaboration's critical assessment of the evidence from RHIC collisions," Nucl. Phys. A757 (2005) 102-183.

[3] B. B. Back et al., "The PHOBOS perspective on discoveries at RHIC," Nucl. Phys. A757 (2005) $28-101$.

[4] BRAHMS Collaboration, I. Arsene et al., "Quark Gluon Plasma an Color Glass Condensate at RHIC? The perspective from the BRAHMS experiment,” Nucl. Phys. A757 (2005) 1-27.

[5] M. Connors, C. Nattrass, R. Reed, and S. Salur, "Review of Jet Measurements in Heavy Ion Collisions," arXiv:1705.01974 [nucl-ex].

[6] STAR Collaboration, H. Agakishiev et al., "Measurements of Dihadron Correlations Relative to the Event Plane in Au+Au Collisions at $\sqrt{s_{N N}}=200 \mathrm{GeV}$," arXiv:1010.0690 [nucl-ex].

[7] STAR Collaboration, B. Abelev et al., "Long range rapidity correlations and jet production in high energy nuclear collisions," Phys.Rev. C80 (2009) 064912.

[8] C. Nattrass, N. Sharma, J. Mazer, M. Stuart, and A. Bejnood, "Disappearance of the Mach Cone in heavy ion collisions," Phys. Rev. C94 no. 1, (2016) 011901. 
[9] PHENIX Collaboration, A. Adare et al., "Measurements of Higher-Order Flow Harmonics in Au+Au Collisions at $\sqrt{s_{N N}}=200 \mathrm{GeV}$," Phys. Rev. Lett. 107 (2011) 252301.

[10] STAR Collaboration, J. Adams et al., "Evidence from d + Au measurements for final state suppression of high p(T) hadrons in Au+Au collisions at RHIC," Phys. Rev. Lett. 91 (2003) 072304.

[11] C. Nattrass, “Jet quenching: an iconic result revisited," arXiv:1801.09131 [nucl-ex].

[12] JET Collaboration, K. M. Burke et al., "Extracting the jet transport coefficient from jet quenching in high-energy heavy-ion collisions," Phys.Rev. C90 no. 1, (2014) 014909.

[13] ATLAS Collaboration, G. Aad et al., "Observation of a Centrality-Dependent Dijet Asymmetry in Lead-Lead Collisions at $\sqrt{s_{N N}}=2.77 \mathrm{TeV}$ with the ATLAS Detector at the LHC," Phys.Rev.Lett. 105 (2010) 252303.

[14] PHENIX Collaboration, A. Adare et al., "High $p_{T}$ direct photon and $\pi^{0}$ triggered azimuthal jet correlations and measurement of $k_{T}$ for isolated direct photons in $p+p$ collisions at sqrts $=200$ GeV," Phys.Rev. D82 (2010) 072001.

[15] CMS Collaboration, S. Chatrchyan et al., "Studies of jet quenching using isolated-photon+jet correlations in $\mathrm{PbPb}$ and $p p$ collisions at $\sqrt{s_{N N}}=2.76 \mathrm{TeV}, "$ Phys. Lett. B718 (2013) 773-794.

[16] ALICE Collaboration, J. Adam et al., "Measurement of jet quenching with semi-inclusive hadron-jet distributions in central Pb-Pb collisions at $\sqrt{s_{\mathrm{NN}}}=2.76$ TeV," JHEP 09 (2015) 170.

[17] ALICE Collaboration, J. Adam et al., “Azimuthal anisotropy of charged jet production in $\sqrt{s_{\mathrm{NN}}}=$ 2.76 TeV Pb-Pb collisions,” Phys. Lett. B753 (2016) 511-525.

[18] STAR Collaboration, G. Agakishiev et al., "System size and energy dependence of near-side di-hadron correlations," Phys.Rev. C85 (2012) 014903.

[19] CMS Collaboration, V. Khachatryan et al., "Correlations between jets and charged particles in $\mathrm{PbPb}$ and pp collisions at $\sqrt{s_{\mathrm{NN}}}=2.76 \mathrm{TeV}$," JHEP 02 (2016) 156.

[20] ATLAS Collaboration, G. Aad et al., "Measurement of inclusive jet charged-particle fragmentation functions in $\mathrm{Pb}+\mathrm{Pb}$ collisions at $\sqrt{s_{N N}}=2.76 \mathrm{TeV}$ with the ATLAS detector," Phys.Lett. B739 (2014) 320-342.

[21] ALICE Collaboration, L. Cunqueiro, "Jet shapes in pp and PbâĂŞPb collisions at ALICE," Nucl. Phys. A956 (2016) 593-596.

[22] ALICE Collaboration, S. Acharya et al., "First measurement of jet mass in PbâĂŞPb and pâĂŞPb collisions at the LHC," Phys. Lett. B776 (2018) 249-264, arXiv:1702.00804 [nucl-ex] .

[23] CMS Collaboration, A. M. Sirunyan et al., "Measurement of the splitting function in pp and $\mathrm{PbPb}$ collisions at $\sqrt{s_{\mathrm{NN}}}=5.02 \mathrm{TeV}, " \operatorname{arXiv}: 1708.09429 \quad$ [nucl-ex].

[24] ALICE Collaboration, N. Zardoshti, "Investigating the Role of Coherence Effects on Jet Quenching in $\mathrm{Pb}-\mathrm{Pb}$ Collisions at $\sqrt{s_{N N}}=2.76 \mathrm{TeV}$ using Jet Substructure," in 26th International Conference on Ultrarelativistic Nucleus-Nucleus Collisions (Quark Matter 2017) Chicago,Illinois, USA, February 6-11, 2017. 2017. arXiv:1705.03383 [nucl-ex].

[25] J. E. Huth et al., "Toward a standardization of jet definitions," in 1990 DPF Summer Study on High-energy Physics: Research Directions for the Decade (Snowmass 90) Snowmass, Colorado, June 25-July 13, 1990, pp. 0134-136. 1990.

[26] G. P. Salam, “Towards Jetography,” Eur.Phys.J. C67 (2010) 637-686. 
[27] T. Sjostrand, S. Mrenna, and P. Z. Skands, "PYTHIA 6.4 Physics and Manual,” JHEP 0605 (2006) 026.

[28] ALICE Collaboration, J. Adam et al., "Measurement of jet suppression in central Pb-Pb collisions at $\sqrt{s_{\mathrm{NN}}}=2.76 \mathrm{TeV}$, , Phys.Lett. B746 (2015) 1-14.

[29] ATLAS Collaboration, G. Aad et al., "Measurements of the Nuclear Modification Factor for Jets in $\mathrm{Pb}+\mathrm{Pb}$ Collisions at $\sqrt{s_{\mathrm{NN}}}=2.76 \mathrm{TeV}$ with the ATLAS Detector," Phys.Rev.Lett. 114 no. 7, (2015) 072302.

[30] J. Novak, K. Novak, S. Pratt, J. Vredevoogd, C. Coleman-Smith, and R. Wolpert, "Determining Fundamental Properties of Matter Created in Ultrarelativistic Heavy-Ion Collisions," Phys. Rev. C89 no. 3, (2014) 034917.

[31] J. E. Bernhard, J. S. Moreland, S. A. Bass, J. Liu, and U. Heinz, “Applying Bayesian parameter estimation to relativistic heavy-ion collisions: simultaneous characterization of the initial state and quark-gluon plasma medium,” Phys. Rev. C94 no. 2, (2016) 024907. 